\title{
A Scheme of Multi-path Adaptive Load Balancing in MANETs
}

\author{
Yang Tao ${ }^{1, a}$, Guochi Lin ${ }^{2, b *}$ \\ ${ }^{1,2}$ School of Communication and Information Engineering, Chongqing University of Posts and \\ Telecommunications, Chongqing, 400065, China

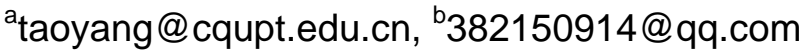

Keywords: MANET, routing protocols, load balancing, AOMDV

\begin{abstract}
The majority of multi-path routing schemes in Mobile Ad-hoc Networks (MANET) does not consider the traffic distribution and load balancing routing performance, increasing network overhead, excessive energy consumption and other issues. We propose a scheme to achieve load balancing when the topology changes rapidly, according to the weights of the path quality to dynamically allocated to the multi-path data flow. The NS2 simulation results indicate that AOMDV-LB is much better than Ad-hoc on-demand multipath distance vector (AOMDV) in network utilization, end-to-end delay performance , and throughput.
\end{abstract}

\section{Introduction}

The network which doesn't require an infrastructure and are dynamically formed using an autonomous system of mobile nodes that are connected via wireless links are known as Mobile Ad Hoc Networks (MANET). The traditional applications of MANETs including battlefield, emergency rescue and data acquisition in remote environment[1]. In MANETs, the node is both a communication terminal and a router. With the constant movement of the nodes, the topology of the network is constantly changing. For the existing MANETs, there are lots of routing protocols, which can be roughly divided into two categories: table driven routing protocol and on-demand routing protocol[2]. In the former, each node in the network needs to maintain and update the routing information continuously. However, in the on-demand routing protocol, not every node needs to maintain the routing information, the nodes can only create the path when the network need to send packets.

In the on-demand routing protocols, the most prominent representations are the AODV protocol and the Dynamic Source Routing (DSR) protocol[3,4]. In this paper, we introduce a simple but efficient method for load balancing. AOMDV provides multiple paths to the destination node, choose the path of the minimum number of hops and discards the higher hop count ones. The new scheme can be applied to most of the on-demand routing protocols, which are used in the routing discovery process. When a route request (RREQ) message[5] is sent to the whole network in a flooding way, not all intermediate nodes receiving the message will broadcast it. The nodes will first compare with a threshold value to determine whether it is overloaded. If the node is overloaded, it will discard the RREQ message, while the newly established path will not contain this overloaded node. The threshold value is used as a criterion, which is dynamically changing and mainly based on the amount of queue occupancy along the reverse path. Throughout this paper, the third part will describe the method of adaptive load balancing in detail. In the fourth part, the performance evaluation and solution is demonstrated in the form of simulation. The fifth part shows the conclusion of this paper.

\section{Related works}

Routing is a fundamental problem in wireless and wired networks so a lot of routing protocols have been proposed[6]. In this section, we will review the existing routing protocols in MANET, especially in terms of load balancing problems. The connection problem is more serious in the single channel networks, because in the single channel network, the connection often occurs when a path passes through the wireless coverage of the other path. This issue has been discussed in the 
literature[7,8]. The load balancing technology in MANETs can be roughly divided into two types, one is based on the flow, which attempt to make equal distribution among the nodes of the network to achieve the load balance; the second is based on time delay, in which the load is balanced by trying to avoid nodes with excessive delay. In this paper, the solution we proposed can be applied in most of on demand routing protocols, both single-path routing and multi-path routing, it is based on the flow type, and will distribute the traffic load evenly among the nodes in MANET.

\section{Multi-path Adaptive Load Balancing Scheme}

AOMDV protocol is an extension of the AODV protocol in order to calculate multiple non loop and link disjoint paths. In order to ensure the existence of the non loop path, if the node already has a path to the destination node, it can only accept an alternate path to the destination if the path has a lower hop count[9]. The adaptive load balancing method we proposed is applied in the process of routing requests. When a source node wants to communicate with the destination node but failed to reach route information to the destination node, it will initialize a route discovery process, then broadcast a RREQ message began to find its way. But not all of the intermediate nodes that receive the RREQ message will respond to it. If the intermediate nodes are qualified to be forwarded, it is first to make a decision before broadcasting the RREQ message again. If its interface queue usage is below the threshold value, the node is qualified and can be broadcast message. Queue occupancy is the number of packets waiting to be forwarded in the interface queue. If the node's interface queue takes up more than the threshold value, the node is not qualified to forward, and at the same time the RREQ message would be discarded. In this way, the overloaded nodes will be excluded from the new path, and according to the on-demand routing protocol, this method will be useful to make the traffic load evenly on the nodes in the network.

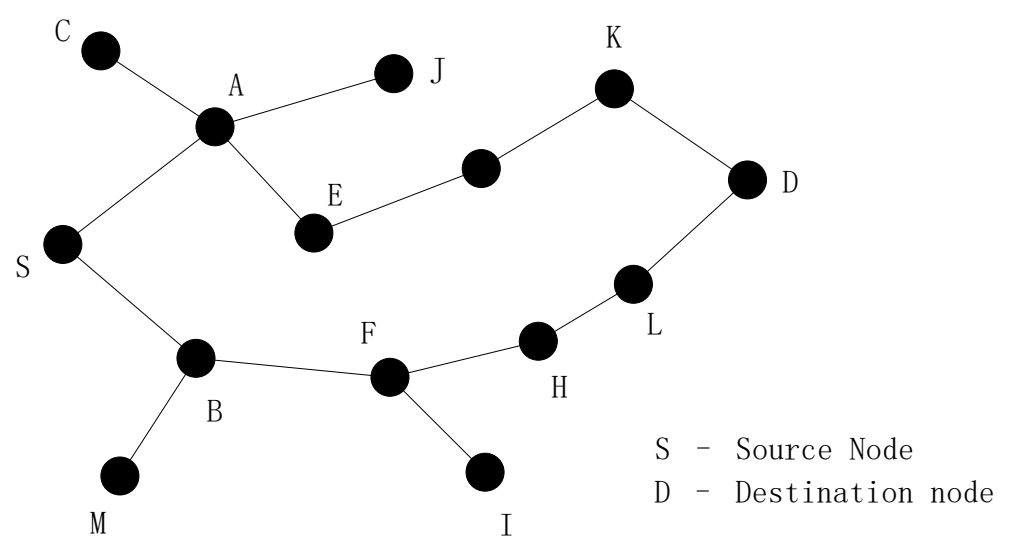

Fig.1. Route Request Process with Adaptive Load Balancing

Through this example we will explain the plan in detail. As shown in Figure 1, the S node is the source node and the $\mathrm{D}$ node is the destination node. When the node $\mathrm{S}$ needs to communicate with the $\mathrm{D}$ node, but it does not have any available path information, then it will initiate a route discovery process by flooding RREQ messages on other nodes of the network. After receiving the RREQ message, the intermediate node determines whether to broadcast or not after checking the current queue capacity and the threshold value. If the queue capacity is greater than the threshold value, the node will directly discarded the RREQ packets, such as nodes J and node I in the figure. Since they do not forward RREQ packets, the newly established path will bypass these nodes. On the contrary, the node will continue to forward RREQ packets, such as nodes A, B, E, etc.. In the above method, the threshold value plays an important role in the selection of nodes which forward the RREQ packets. Once the intermediate node receives a RREQ packet, it will recalculate the threshold value which is based on the queue capacity of the nodes on the reverse path. Therefore, the threshold value is variable, and it will make the adaptive change according to the current load condition of the network. Here we give an algorithm to calculate the threshold value of each node. 
Step 1:The node will calculate the average queue occupancy ( $a v g \_q o c$ ) based on the current queue occupancy of all nodes in the local area. Therefore, the node's avg _ qoc can be calculated according to the following formula:

$$
a v g_{-} q O c=\frac{q O c+\sum_{i=1}^{n} n b_{-} q o c_{i}}{n+1}
$$

Here qoc represents the queue occupancy of the node itself, $n b_{-} q o c_{i}$ is the queue occupancy of the neighbor nodes, and $n$ is the number of neighbors of the node. As shown in Figure 1, the node A's average queue occupancy can be determined using its own queue occupancy, and its neighbor nodes $\mathrm{C}, \mathrm{S}, \mathrm{E}$ and $\mathrm{J}$ queue occupancy, $n b_{-} q o c_{1}, n b_{-} q o c_{2}, n b_{-} q o c_{3}$ and $n b_{-} q o c_{4}$. Here $n$ is equal to 4 . The source node will calculate the average queue occupancy by avg _qoc .

Step 2: the threshold value of the node (thr) will be calculated based on the average queue occupancy of all nodes on the path.

By the node's own avg _ qoc and the RREQ's sum_qoc it received along the nodes on the reverse path, the node $\mathrm{G}$ will be able to compute the threshold value according to its $a v g$ _ qoc and the received RREQ packets' sum_qoc, which is the sum of $a v g \_q o c$ of node $\mathrm{S}, \mathrm{A}, \mathrm{E}$.

$$
\begin{aligned}
& \bar{d}=\frac{\left|q o c-a v g_{-} q o c\right|+\sum_{n=1}^{n}\left|n b_{-} q o c_{i}-a v g_{-} q o c\right|}{n+1} \\
& \text { thr }=\bar{d}+a v g_{-} q o c
\end{aligned}
$$

Step 3: the node compares its current queue occupancy ( $q 0 c$ ) and the threshold value (thr ). When the node gets its own thr, it will compare the value with the current qoc, if the threshold value is greater than qoc , the node will respond to the RREQ packet. Otherwise, it will discard it.

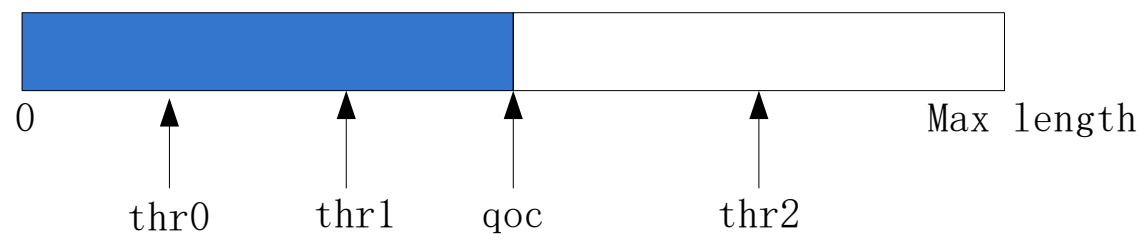

Fig.2. Comparing the qoc with thr

As shown in Figure 2, if $t h r=t h r 1$, then $q o c>t h r$, the received RREQ packets will be discarded; if $t h r=t h r 2$, then $q o c<t h r$, the received RREQ packets will be continued to broadcast; if $t h r=0$, then $t h r=t h r 0$.

Solution: the following presentation of the program is the changes to the existing AOMDV protocol.

1.Paths are selected according to the hop count and queue length.

2.If the queue length is within a certain threshold value, the load balance will be achieved by making the choice of the paths.

3. Whether the RREQ packets are forwarded or dropped is according to the queue length of the node. 


\section{Simulation and results}

In this part, we use our load balancing method in AOMDV, which called AOMDV-LB, then we evaluate the performance analysis of the two algorithms in the simulation environment.

AOMDV is an on-demand multi-path distance vector routing protocol that select a path with the minimum hop count, while discarding the paths with higher hop count.

Simulation environment: This paper uses the NS-2 simulation environment, the simulation parameters we set are: node evenly distributed in the $800 \mathrm{~m} * 800 \mathrm{~m}$ rectangular area, a total of 25 mobile nodes within the transmission distance of $250 \mathrm{~m}$. The bandwidth of the network is $2 \mathrm{Mbps} / \mathrm{s}$, and the Mac layer protocol uses 802.11. The number of destination nodes is 1 .

Simulation uses a fixed bit rate for the traffic, it transmits 5 packets per second and the size of each packet is 512 bytes.

Simulation result analysis: In the above simulation environment, the follow three performance will be evaluated and analyzed: network loss ratio, end to end delay, load distribution.

1.Network loss ratio: The ratio of the number of lost packets and the total number of packets transmitted during the data transmission, is used to measure the congestion of the network.

2.Average end-to-end delay: it refers to the time taken for a packet to be transmitted across the network from source to destination, which is used to measure the routing efficiency.

3.load distribution: it aims to optimize resource use, maximize throughput, minimize response time, and avoid overload of any single resource.

In this paper, we set the initial threshold value equal to 5 packets. In this case, the majority of the node's queue occupancy will not be more than 5 packets.

Figure 3 shows the network loss ratio. When the network is in a low load environment, the network loss ratio of AOMDV-LB has little difference between with the AOMDV. With the increasing of the load in the network, the network loss ratio of AOMDV-LB and AOMDV has been increased, but the loss rate of the former is little than the latter. Therefore, the network loss ratio in the AOMDV-LB is significantly reduced, and the performance is improved.

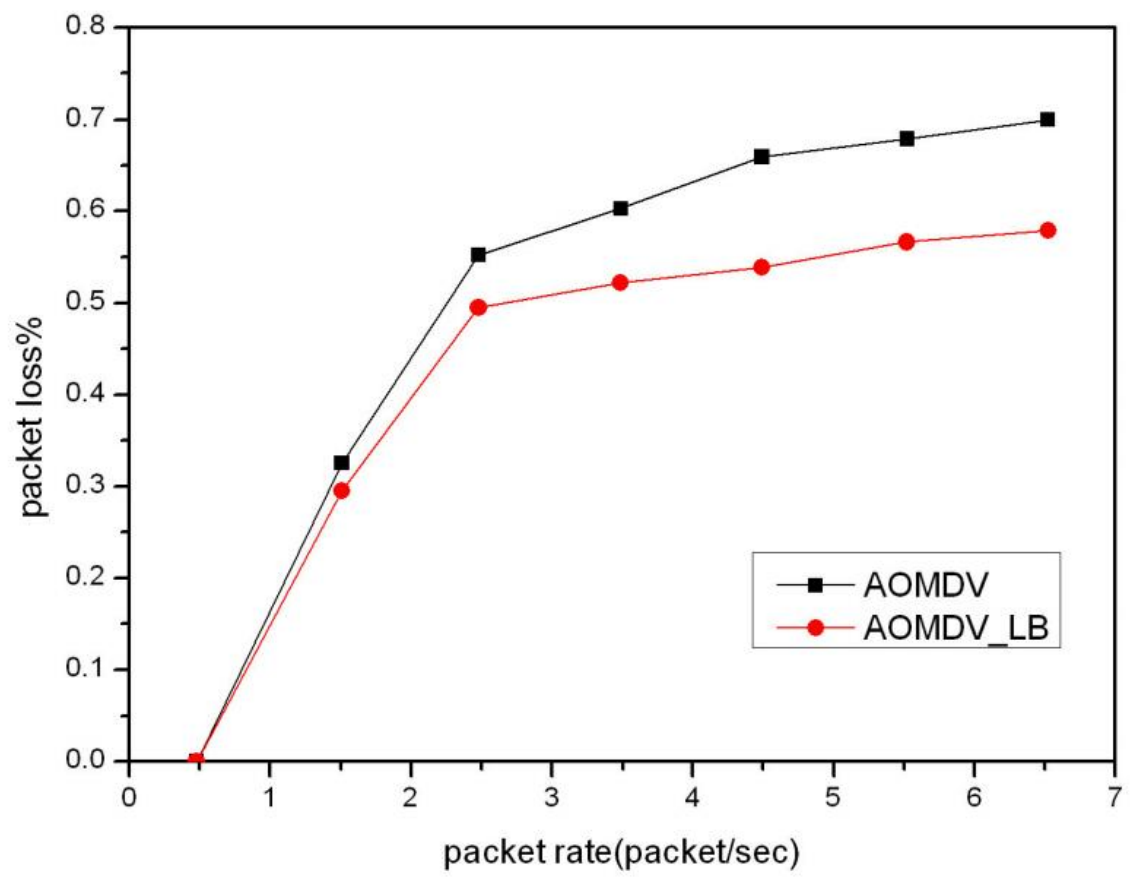

Fig.3. Network Loss Ratio

Figure 4 shows the average end to end delay of the transmission data. When the traffic load in the network increases, the end-to-end delay will increase, which is mainly caused by the congestion. AOMDV-LB can find the path with low traffic load, but the AOMDV shows a poor performance 
when the network load is serious. Therefore, in the AOMDV-LB the average end to end delay are significantly reduce.

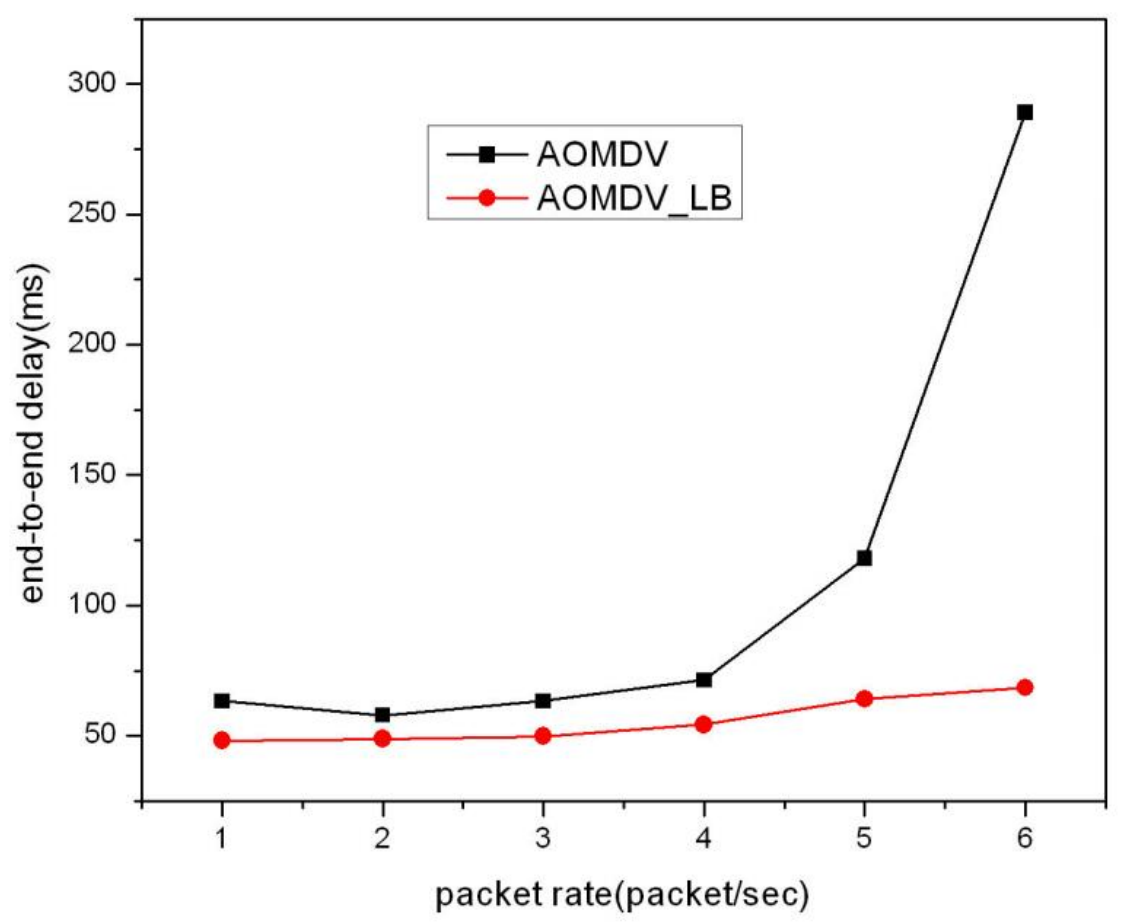

Fig.4. Average End-to-end Delay

Figure 5 shows the node's load distribution graph. The forwarded packets size in the AOMDV nodes have much more difference between each other and the network load is not balanced. However, the data packet size forwarded in the AOMDV-LB is relatively stable, it is due to the reasonable allocation of the flow in the network and can achieve the load balance. Therefore, the load distribution is more balanced in the AOMDV-LB.

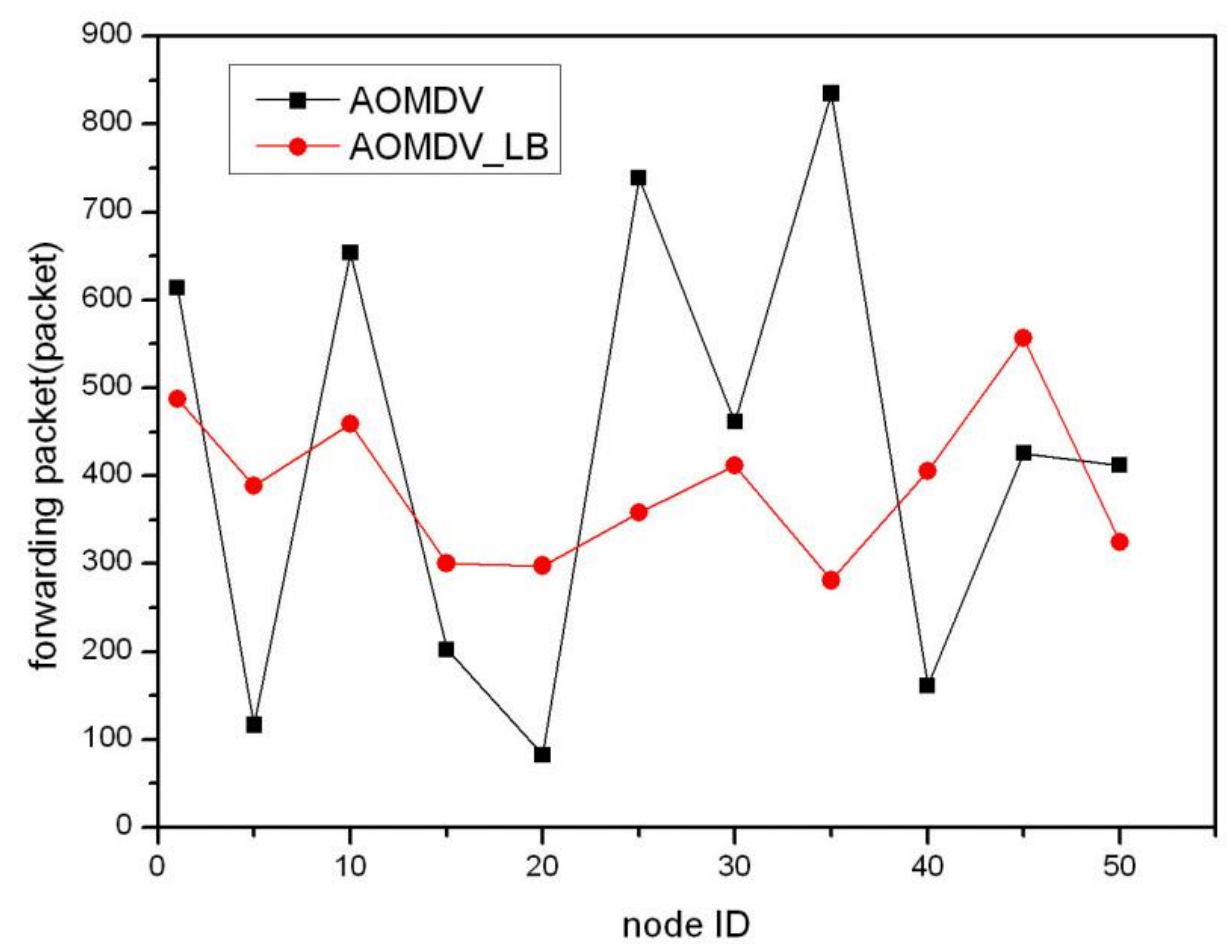

Fig.5. Load Distribution 


\section{Conclusion}

In this paper, each node determines how to response to the received RREQ packet by examining its interface queue occupancy. The criterion is the threshold value, which is calculated after the RREQ packet is received at each node. It can dynamic change with the change of the queue occupancy of each node along the reverse path. Therefore, the threshold value will be adjusted according to the traffic load in the network. This program can make the traffic in the MANETs distribute evenly to the network nodes.

\section{References}

[1] Sarkar S K, Basavaraju T G, Puttamadappa C. Ad hoc mobile wireless networks: principles, protocols and applications[M]. CRC Press, 2007.

[2] Bai M R, Ramesh V, Kumar D V. Design of Load Balanced Ad-hoc on Demand Multi Path Distance Vector Routing for MANETs[J]. Journal of Network Communications and Emerging Technologies (JNCET) www. jncet. org, 2015, 2(3).

[3] Liu C X, Zhang Y, Xu E, et al. A Novel Multi-Path Routing Algorithm Based on Clustering for Wireless Mesh Networks[J]. KSII Transactions on Internet and Information Systems (TIIS), 2014, 8(4): 1256-1275.

[4] Thaalbi M, Tabbane N, Bejaoui T, et al. A weighted QoS aware multipath routing process in Mobile Ad hoc Networks[C]//Computers and Communications (ISCC), 2013 IEEE Symposium on. IEEE, 2013: 000336-000341.

[5] Guo L, Zhang L, Peng Y, et al. Multi-path routing in spatial wireless ad hoc networks[J]. Computers \& Electrical Engineering, 2012, 38(3): 473-491.

[6] Yang P, Huang B. QoS routing protocol based on link stability with dynamic delay prediction in MANET[C]//Computational Intelligence and Industrial Application, 2008. PACIIA'08. Pacific-Asia Workshop on. IEEE, 2008, 1: 515-518.

[7] Abolhasan M, Wysocki T, Dutkiewicz E. A review of routing protocols for mobile ad hoc networks[J]. Ad hoc networks, 2004, 2(1): 1-22.

[8] Devi P R, Rao D D S. Congestion Adaptive Hybrid Multi-path Routing Protocol for Load Balancing in Mobile Ad Hoc Networks[J]. International Journal of Computer Science and Telecommunications, 2012, 3(12): 2345-4356.

[9] Attar M A, Edsall T J, Dharmapurikar S M, et al. System and method for multi-path load balancing in network fabrics: U.S. Patent Application 14/308,464[P]. 2014-6-18. 